comparison of the number of false-recognition errors to $R$ words vs $C R$ words and to $S$ words vs $C S$ words under the two timing conditions. It may be seen that all comparisons are significant (one just barely misses the .05 mark). Because of the inordinately low error rate for the CSs in the 2 -sec condition, a direct statistical comparison between the two conditions seems inappropriate. But inspection of Table 1 makes it clear that the two conditions did not differ in the total number of false-recognition errors or in the relative number of $S$ and $R$ errors. In fact, the total percentage of errors for all 220 words, including both false-recognition errors and false-nonrecognition errors (saying "no" to old words) was virtually identical for the two conditions, $2-\sec =7.4 \%, 5-\sec =7.5 \%$. The failure to obtain a difference between the two conditions does not necessarily mean that the time factor plays no role in a recognition task of this sort. As an altemative, it appears quite reasonable to assume that $S s$ in the 5 -sec condition did not use the extra time available to them in a task-relevant manner. Since each word was both a memorization and a test item, it does not seem unlikely that Ss focused attention primarily on the test aspect and, after giving their responses, let the item slip out of their minds in anticipation of the next (test) item. Also, the time interval between presentation of the item and S's re sponse does not appear, from listening to the tapes, to be appreciably longer in the 5 -sec condition than in the $2-\sec$ condition. This interpretation is supported by Ss' comments given in a postexperimental interview. Most of the Ss in both conditions said they did not rehearse or think about a word after giving a response to it.

In both conditions, both semantic and phonetic relations produced false-recognition errors. Why? The phenomenon of false recognition was interpreted earlier (Anisfeld \& Knapp, 1968; see also Anisfeld, 1967) as suggesting that words are not coded wholistically but in terms of the composite of semantic, syntactic, phonetic, and orthographic features characteristic of them. When a word is heard, (some of) these features are activated, and their traces persist in a more or less marked state for some time. False recognition arises when a later word that shares some features with a previous word is presented. The finding that the rhyming relations were as effective in producing false-recognition errors as the semantic relations suggests that the traces of the phonetic features on which rhyming depends retained their marked state as long as the semantic traces did. This finding is in accord with the results of Felzen \& Anisfeld (in press), but at variance with Wallace's
(1968) study, which, it will be recalled, obtained no false-recognition errors for phonetically related words uncer conditions of full attention. The difference in results may be attributable to a difference in the time separation between the $P$ words and the semantically and phonetically related words. The maximum separation in the present experiment was $5 \mathrm{~min}$, and in Wallace's experiment, the minimum separation was $17 \mathrm{~min}$. The longer time may have caused the traces of the phonetic features to fade. This interpretation suggests that phonetic traces fade faster than semantic traces.

\section{REFERENCES}

ANISFELD, M. Review of J. Deese, Associations in language and thonght. American Journal of Psychology, 1967, 80, 646-649.

ANISFELD, M., \& KNAPP, M. E. Association, synonymity, and directionality in false recognition. Journal of Experimental Psychology, 1968, 77, 171-179.

EAGLE, M., \& ORTOF, E. The effect of level of attention upon "phonetic" recognition errors. Journal of Verbal Learning \& Verbal Behavior $1967,6,226-231$.
FELZEN, E., \& ANISFELD, M. Semantic and phonetic relations in the false recognition of words by third- and sixth-grade children. Developmental Psychology, in press.

PALERMO, D. S., \& JENKINS, J. J. Word association norms; grade school through college. Minneapolis: University of Minnesota Press, 1964.

THORNDIKE, E. L., \& LORGE, I. The teacher's word book of 30,000 words. New York: Teachers College, Columbia University, 1944. UNDERWOOD, B. J. False recognition produced by implicit verbal responses. Joumal of Experimental Psychology, 1965, 70, 122-129. WALLACE, W. P. Incidental learning: The influence of associative similarity and formal similarity in producing false recognition. Journal of Verbal Learning \& Verbal Behavior, $1968,7,50-54$.

\section{NOTES}

1. Part of the impetus for this research came from discussions with $U$. Neisser. J. Gall and $K$. Sharon helped in the experiment. This research was supported by Grant GB-13342 from the National Science Foundation.

2. Present address: Department of Psychology, Yeshiva University, New York, N.Y. 10003.

\title{
Sex differences in the development of fearfulness:
} \section{A replication}

\section{GORDON W. BRONSON, Mills College, Oakland, Calif. 94613}

Human infants differ in the age at which they first begin to-fear the visually unfamiliar. Among a sample of male infants, a precocious onset of fear was predictive of heightened fearfulness at age 1 year; such developmental continuity was not observed in a comparable female sample. Almost identical patterns were observed in a previous sample; the present replication strengthens the argument for a sex-linked constitutional factor that contributes to the ontogeny of fearfulness.

Although the age of onset will vary somewhat, most human infants begin to be wary of the visually unfamiliar during the middle half of their first year of life. A previous study (Bronson, in press) established that male infants in whom such fear reactions appeared relatively early were significantly more shy of strangers during infancy and into the preschool years; for female infants, however, no such patterns could be established. The present report replicates this finding of a sex difference in developmental continuity in a second sample of human infants.

\section{PROCEDURE}

The data were obtained from Ainsworth's longitudinal study, in which 24 infants were observed in their homes at intervals of 3 weeks throughout the 1st year of life and then tested in an experimentally controlled "strange situation" at age 1 year. 1

The age of onset of fear was inferred from descriptions of the infants' behavior toward the $O$ during the periodic home visits. Original protocols were rated on two scales devised to codify behaviors indicative of fear: Scale $A$ described the infant's smiling behavior toward the $O$, from (1) "Smiled almost immediately" to (5) "Gave no smile to O"; Scale B reflected other indices of fearfulness, from (1) "Showed no evidence of wariness" to (5) "Cried when 0 approached." The distinction between Points 2 and 3 on Scale B deserves special comment. Point 2 was defined as "Looked repeatedly at $O$, but did not seem inhibited by his presence"; Point 3, "Seemed wary of 0 : stared, quieted, for a considerable period." Since infants often show an intense interest in the visually unfamiliar some time before the onset of behavior that can be interpreted as fearful (see Ainsworth, 1967, and Schaffer \& Parry, 1969), only 

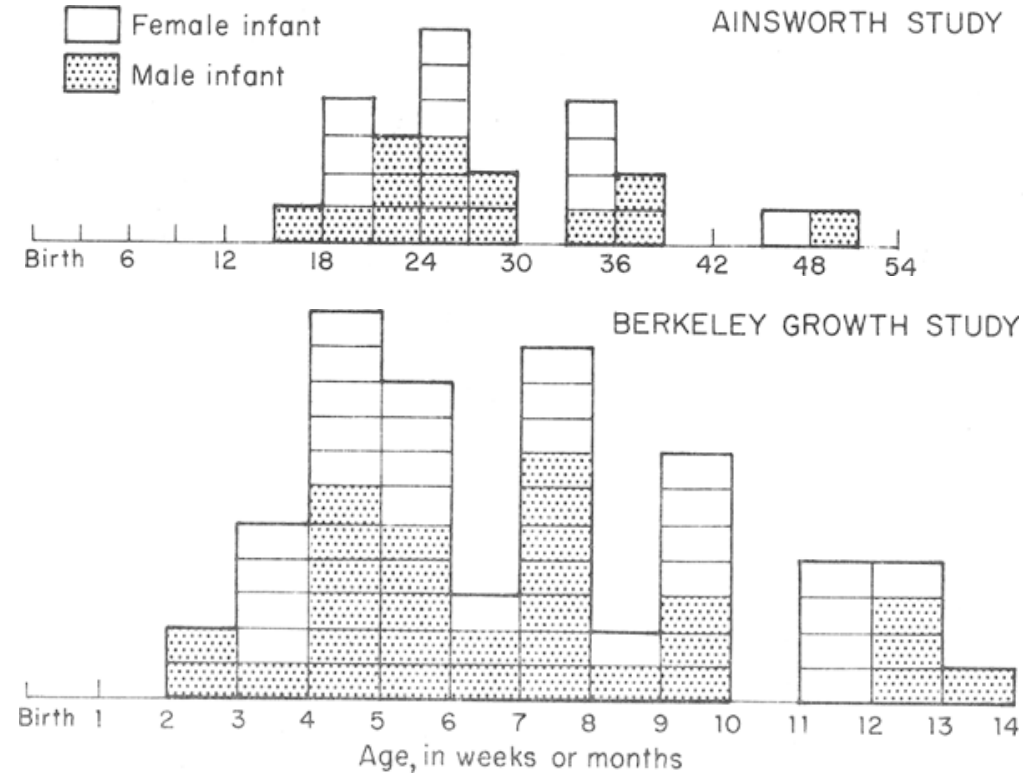

scores of 3 or greater were taken as evidence of fear. Scores on Scales A and B were found to be highly correlated; the age of onset of fear was defined as the first age when an infant's average score on the two scales reached or exceeded a value of 3 .

The intensity of an infant's fear at age 1 year was determined from behavior in the "strange situation." The situation consisted of a series of eight 3-min episodes occurring in an unfamiliar room that contained an assortment of playthings; the mother and stranger were variously present or absent in the different episodes. Only behavior in Episode 3 was used for the present study since the remaining episodes in which the stranger was present involved separation from the mother, thus confounding reactions to novelty with separation anxiety (for details of procedures in the various episodes, see Ainsworth \& Wittig, 1969). Episode 3 began with the entrance of a strange person (S). For the first half of this episode, $\mathrm{S}$ remained seated, quietly talking with the mother; Scale C reflected infants' reactions during this interval, from (1) "Noted presence of $S$ but continued to play" through (5) "Cried and returned to mother." Midway through this episode, S approached and attempted to engage the infant in play. Scale D coded infants' reactions to S's approach, from (1) "Engaged in play with $S$ " through (5) "Cried at S's approach." Scores on the two scales were averaged to provide an index of the intensity of fear in the presence of a stranger.

Two judges independently coded the infants' behaviors during the home observations and in the "strange situation"; removal of all identification from protocols insured independence of the two sets of judgments. Interrater agreement was high; out of a total of 257 pairs of ratings made in all the four scales, $79 \%$ were in complete agreement, and $98 \%$ were within one scale point.

\section{RESULTS AND DISCUSSION}

The degree of correlation between age of onset of fear and the intensity of fear reactions at age 1 year was computed separately for males and females. The correlation for boys was $\mathrm{r}=-.48(\mathrm{~N}=14$; one-tailed $p=.05) ;$ for girls, $r=.05$ $(\mathrm{N}=10$, n.s.). For males-but not for females-an early onset of fear was significantly related to a heightened fearfulness at age 1 year.

Comparable indices computed for Ss of the previous study, based on data from the Berkeley Growth Study (see Bronson, in press), proved to be almost identical: The relation between age of onset of fear and degree of shyness toward a strange examiner at ages 10 to 15 months for boys was $r=-.46(N=31, p=.01)$; for girls, $\mathrm{r}=-.06(\mathrm{~N}=27, \mathrm{n.s}$.$) . It is noteworthy$ that in both studies the distribution of cases within the scatter-plots for male Ss indicate that factors other than error variance attenuated the observed correlations. In both instances, all the male infants who fell considerably outside of a linear pattern appeared in the same quadrant; these Ss showed a relatively early onset of fear yet, in contrast to the general pattern, were not unusually fearful at the end of the 1st year. Factors that might account for this consistent deviant pattern remain to be identified.

Although the procedures for assessing age of onset of fear differed considerably in the present and in the previous study,
Fig. 1. Age of onset of fear of the unfamiliar, as observed in two longitudinal studies.

the observed age distributions are almost identical. Figure 1 presents these distributions; note that they fail to conform with the finding of Robson, Pederson, \& Moss (1969) that females, on the average, develop fear reactions earlier than do males.

The fear of novelty is sometimes noted as "8-months anxiety," and, indeed, the frequency of occurrence within a sample of infants probably does increase toward the end of the 1st year (see Bronson, 1968). It seems clear, however, that infants differ widely in the age of first appearance of this developmental event, and that for many infants it occurs considerably before age 8 months; in addition, the predictive value of a relatively early or late onset differs for male and female infants. A careful analysis of pattems found in the Berkeley Growth Study indicates that this latter effect cannot easily be explained by reference to a presumed difference in mothers' behaviors toward male and female infants. The present replication of sex differences in developmental patterns lends further support to the thesis that sex-linked constitutional differences interact with experiential factors in determining the ontogeny of fearfulness in early childhood (see Bronson, in press).

\section{REFERENCES}

AINSWORTH, M. D. S. Infancy in Uganda Baltimore: Johns Hopkins Press, 1967. Pp. 358-359.

AINSWORTH, M. D. S., \& WITTIG, B. A. Attachment and exploratory behavior of one-year-olds in a strange situation. In B. M. Foss (Ed.), Determinants of infant behavior, $I V$. London: Methuen, 1969. Pp. 111-136.

BRONSON, G. W. The development of fear in man and other animals. Child Development, $1968,39,409-431$.

BRONSON, G. W. Fear of visual novelty: Developmental patterns in males and females. Developmental Psychology, in press.

ROBSON, K. S., PEDERSEN, F. A., \& MOSS, H. A. Developmental observations of diadic gazing in relation to the fear of strangers and social approach behavior. Child Development, $1969,40,619-628$.

SCHAFFER, H. R., \& PARRY, M. H. Perceptual-motor behaviour in infancy as a function of age and stimulus familiarity. British Journal of Psychology, 1969, 60, 1-9. NOTE

1. I deeply thank Dr. Mary Ainsworth for her generosity in allowing me to analyze data from her study. Support for her research came from The Foundation's Fund for Research in Psychiatry, Grant 62-244, and from USPHS Grant RO1 HD01712. Data from the Berkeley Growth Study was made available through the courtesy of Dr. Nancy Bayley. The research was supported by NIMH Grant MH 08135 to the University of California Institute of Human Development. 